\title{
Clinical Tools for Peripheral Neuropathy to Exclude Cardiovascular Autonomic Neuropathy in Type 2 Diabetes Mellitus
}

Kalliopi Pafili · Grigorios Trypsianis · Dimitrios Papazoglou •

Efstratios Maltezos $\cdot$ Nikolaos Papanas

Received: January 29, 2020 / Published online: March 14, 2020

(C) The Author(s) 2020

\section{ABSTRACT}

Introduction: Assessment for cardiovascular autonomic neuropathy (CAN) remains difficult in everyday clinical practice. We sought to examine the diagnostic utility of various simple tools for diabetic peripheral neuropathy (DPN) in the detection of CAN in type 2 diabetes mellitus.

Methods: We examined 153 type 2 diabetes mellitus subjects by various DPN tools (vibration perception threshold, $10 \mathrm{~g}$ Semmes-Weinstein monofilament, Ipswich touch test, NCstat ${ }^{\circledR} /$ DPNCheck, neuropathy disability score) for the detection of CAN. CAN was diagnosed

Digital Features To view digital features for this article go to https://doi.org/10.6084/m9.figshare.11907234.

K. Pafili $(\bowtie) \cdot$ D. Papazoglou · E. Maltezos ·

N. Papanas

Diabetes Centre, Second Department of Internal Medicine, University Hospital of Alexandroupolis, Democritus University of Thrace, Alexandroupolis, Greece

e-mail: kpafili@hotmail.com

G. Trypsianis

Department of Medical Statistics, Medical Faculty, Democritus University of Thrace, Alexandroupolis, Greece by the standardised cardiovascular autonomic reflex function tests.

Results: For the diagnosis of CAN, assessment of small nerve fibre function (pinprick sensation, temperature perception) yielded a very high negative predictive value (97\%), with high sensitivity (89\%) and moderate specificity (73\%). The vibration perception threshold was second in diagnostic utility (91\% negative predictive value, $62 \%$ sensitivity and $75 \%$ specificity).

Conclusions: Based on their high negative predictive value, simple tools for DPN may prove useful to exclude CAN in type 2 diabetes mellitus. These encouraging results merit further evaluation to enable wider screening for CAN.

Keywords: Cardiac autonomic neuropathy; Diagnosis; Ewing's battery; Somatic neuropathy 


\section{Key Summary Points}

Why carry out this study?

In type 2 diabetes mellitus (T2DM), improved screening for cardiovascular autonomic neuropathy (CAN) is still needed.

In this setting, we examined the diagnostic utility of various simple tools for diabetic peripheral neuropathy (DPN) in the detection of CAN in T2DM.

We tried various DPN tools (vibration perception threshold, $10 \mathrm{~g}$ SemmesWeinstein monofilament, Ipswich touch test, NC-stat ${ }^{\circledR} /$ DPNCheck, neuropathy disability score).

\section{What was learned from this study?}

The best diagnostic performance was yielded by: (1) the assessment of small nerve fibre function (pinprick sensation, temperature perception): $97 \%$ negative predictive value, $89 \%$ sensitivity and $73 \%$ specificity; (2) the vibration perception threshold: $91 \%$ negative predictive value, $62 \%$ sensitivity and $75 \%$ specificity.

In view of their high negative predictive value, simple tools for DPN may prove useful to exclude CAN in T2DM.

\section{INTRODUCTION}

Cardiovascular autonomic neuropathy (CAN) is defined as the impairment of cardiovascular autonomic control in humans with diabetes mellitus after exclusion of other causes [1, 2]. CAN may lead to life-threatening complications including coronary ischaemia, silent myocardial infarction, arrhythmias and sudden cardiac death $[1,2]$.

Cardiovascular autonomic reflex function tests (CARTs), as proposed by Ewing in 1970, are considered the gold standard for the diagnosis and staging of CAN [3]. Nonetheless, new evidence has shown a diminished diagnostic utility of the handgrip test, and so it has been abolished by expert groups in the most recent guidelines [4]. Even by performing four instead of five tests, the so-called "Ewing's battery" remains a time-demanding approach and depends on patient co-operation. In view of these limitations, previous studies have aimed to simplify the diagnosis of CAN in type 2 diabetes mellitus (T2DM) [5-9]. However, they did not eliminate the need for special equipment and trained personnel [5-9]. Thus, a simple, noninvasive, easily available screening test for CAN is still required.

Conversely, diagnosis of diabetic peripheral neuropathy (DPN) is based on simple bedside diagnostic tools [10-12]. Among these, the neurothesiometer, the $10 \mathrm{~g}$ Semmes-Weinstein monofilament and Ipswich touch test assess large nerve fibre impairment $[10,11]$. The portable $\mathrm{NC}$-stat ${ }^{\circledR} / \mathrm{DPNCheck}{ }^{\mathrm{TM}}$ device also assesses large fibre function (automated sural nerve conduction study) $[13,14]$. Temperature and pain perception assess small fibre function $[1,3]$. Overall, the established Neuropathy Disability Score (NDS) is a clinical examination score reliably assessing both small and large fibre function $[1,3]$.

Therefore, the aim of the present study was to examine the diagnostic utility of various simple and affordable tools for DPN to detect CAN in individuals with T2DM.

\section{METHODS}

\section{Patients}

This study included 153 T2DM participants (92 men, 61 women) with mean age of $64.4 \pm 7.8$ years and mean T2DM diabetes duration of 12 years (1-34 years). CAN was present in 26 individuals (17\%) and DPN in 53 individuals (34.6\%). All participants attended the Diabetes Centre of the Second Department of Internal Medicine at Democritus University of Thrace, Greece. This study received approval from the institutional ethics committee. The study was conducted in accordance with the 
Declaration of Helsinki 1964 and its later amendments. Informed consent was obtained from all participants of this study.

Participants were selected randomly to avoid selection bias. The following procedure was used, as described earlier [12]: Two T2DM persons who were about to attend a scheduled appointment in the Diabetes Centre were chosen randomly by a member of the administrative staff and were informed prior to their appointment on the purpose of the study.

Exclusion criteria were as follows: age $\geq 85$ years, mental illness, inability to complete the examination and other causes of neuropathy $[15,16]$. In addition, patients with proliferative retinopathy were excluded from the Valsalva examination [2]. Age $\geq 85$ years was chosen among exclusion criteria because autonomic function responses have shown a significant decline with increasing age and because agerelated values of the expiration to inspiration ratio assessed during heart rate variation with deep breathing do not apply for individuals aged $>$ 85 years $[2,5]$.

\section{Assessment of CAN}

CAN was assessed by the four standardised CARTs as described by Ewing in 1970: heart rate variation with deep breathing with assessment of expiration to inspiration $(E / I /)$ ratio, heart rate analysis in the standing position (the 30:15 ratio), the Valsalva ratio and the postural change of blood pressure $[2,5]$. Each examination took place early in the morning (07:00-09:00 a.m.) in a quiet environment and at a steady room temperature $\left(22-24^{\circ} \mathrm{C}\right)$. Participants were instructed to avoid food and particular pharmacological agents (antidepressants, neuroleptics, nicotine and caffeine) for the $12 \mathrm{~h}$ preceding the examination. Participants were further requested to avoid insulin and hypoglycaemic agents on the day of the procedure [12].

Examination was carried out with the use of the computer-aided system Varia cardio TF5 (MIE Medical Research, Leeds, UK) [12]. An E/ $I$ ratio above the age-related reference value, a Valsalva ratio $\geq 1.21$, a posture ratio $\geq 1.04$ and a systolic blood pressure reduction in response to standing $\leq 10 \mathrm{mmHg}$ were considered normal [12]. An E/I ratio below the age-related values, a Valsalva ratio $\leq 1.10$, a posture ratio $\leq 1.00$ and a systolic blood pressure fall in response to standing $\geq 20$ were considered abnormal. Each of the items was scored as 0 for normal, 1 for borderline, and 2 for abnormal. CAN was defined as $\geq 2$ abnormal tests $[2,5]$.

\section{Assessment of DPN: Large Nerve Fibres}

Large nerve fibre function was assessed by a variety of diagnostic tools [15-21]. Vibration perception threshold (VPT) was measured with a neurothesiometer (Horwell Scientific Laboratory Supplies, London, UK) on the pulp of the hallux. Given the low sensitivity of the $25 \mathrm{~V}$ threshold for early detection of DPN [17], the lower threshold of $\geq 16 \mathrm{~V}$ was preferred [18].

Moreover, the 10-g Semmes-Weinstein monofilament was applied in an arrhythmic manner at ten sites of each lower extremity: the distal part of the great toe, third and fifth toe, first, third and fifth metatarsal bones, medial foot, lateral foot, heel and dorsally between the great and second toe. Sites with ulcer, scar, callus and necrotic tissue were avoided $[19,20]$. Inability to perceive at least two sites in at least one lower extremity was defined as abnormal [20].

Examination further included the Ipswich touch test [21]. The examiner applied mild pressure with the index finger for approximately $1-2 \mathrm{~s}$ in the apex of the first, third and fifth toe of each lower limb. Inability to perceive at least two touches (out of the six overall for the two lower extremities) was characterised abnormal [21].

The portable, non-invasive NC-stat ${ }^{\circledR} /$ DPNCheck TM device (NeuroMetrix, Inc., Waltham, MA) $[13,14]$ was used for automated sural nerve conduction study. Examination was considered abnormal when amplitude was $<4 \mu \mathrm{V}$ and/ or when conduction velocity was $<40 \mathrm{~m} / \mathrm{s}$ in at least one of the two lower extremities [14]. In this approach, we did not use absolute values of amplitudes and/or nerve conduction velocities. Instead, a qualitative evaluation of $\mathrm{NC}^{-}$stat $^{\circledR} /$ DPNCheck ${ }^{\mathrm{TM}}$ data (normal or abnormal result) was applied, as in a previous study [14]. 
Finally, Achilles reflexes and vibration perception with the use of a $128-\mathrm{Hz}$ graded tuning fork at the apex of the hallux were separately assessed as the large nerve fibre components of NDS [16]. The test was considered positive when at least one of these two parameters was abnormal in at least one foot.

\section{Assessment of DPN: Small Nerve Fibres}

The small fibre NDS components pinprick sensation and temperature perception (with a Tiptherm rod) were used [22]. The test was considered positive for small nerve fibre impairment when at least one of the two parameters was abnormal in at least one lower extremity.

\section{Overall DPN Assessment: Both Large and Small Nerve Fibres}

Finally, DPN was assessed with the use of NDS [23]. Three parameters (pinprick sensation, temperature perception and vibration perception threshold) were scored as normal (0) or abnormal (1), whereas Achilles reflexes were scored as present $(0)$, present with reinforcement (1) and absent (2). A sum of components $\geq 3$ was considered diagnostic of DPN [23].

\section{Statistical Analysis}

Statistical analysis of the data was performed using the Statistical Package for the Social Sciences (SPSS, Chicago, IL), version 19.0. Normality of quantitative variables was tested with the Kolmogorov-Smirnov test. Normally distributed quantitative variables were expressed as the mean \pm standard deviation (SD), while non-normally distributed quantitative variables were expressed as median and range ( $\mathrm{min}$ to max). Categorical variables were expressed as frequencies (and percentages). Sensitivity, specificity, positive (PPV) and negative predictive values (NPV) were calculated, while Cohen's kappa was used to assess agreement. All tests were two-tailed and significance was defined at the $5 \%$ level $(p<0.05)$.

\section{RESULTS}

Table 1 shows the diagnostic accuracy of the various diagnostic tools for DPN in the diagnosis of CAN. Assessment of small nerve fibre function with the use of NDS (pinprick sensation, temperature perception) yielded the best NPV (97\%) for the diagnosis of CAN, with high sensitivity (89\%), moderate specificity (73\%) and PPV $40 \%$. VPT exhibited the second best NPV of $91 \%$, with a moderate sensitivity (62\%) and specificity (75\%). Table 1 also shows the overall agreement of patient classification according to the various tools for DPN with CAN diagnosis. This exceeded $65 \%$ for all diagnostic tools, except for large nerve fibre function evaluation by NDS. However, Cohen's coefficient kappa failed to exceed moderate agreement, with one exception: assessment of small nerve fibre function by NDS (Cohen's kappa coefficient 0.418).

\section{DISCUSSION}

This study has shown that assessment of small nerve fibre function (pinprick and temperature sensation) yielded an extremely high NPV (97\%), with high sensitivity (89\%) and moderate specificity (73\%) for the diagnosis of CAN. $\mathrm{VPT} \geq 16 \mathrm{~V}$ was second in diagnostic utility with a high NPV of $91 \%$ and an adequate sensitivity (62\%) and specificity (75\%). Overall agreement of patient classification according to the different DPN diagnostic tools was $>65 \%$ for all tools, except for the evaluation of large nerve fibre function by NDS. Hence, based on the very high NPV shown in our results, it appears that simple bedside tools recommended by current guidelines for the diagnosis of DPN [16] may prove useful to exclude CAN.

Previous studies have also attempted a simplification of CAN diagnosis through the use of DPN diagnostic tools. These primarily focused on evaluation of the diagnostic performance of sudomotor function (i.e. sweating) [12, 23-26]. In a mixed population including 45 individuals with type 1 diabetes mellitus (T1DM) and 25 healthy volunteers, the sensitivity and specificity of Sudoscan (Impeto Medical, Paris, 
Table 1 Diagnostic performance of DPN tools for the diagnosis of CAN

\begin{tabular}{lllllll}
\hline & Sensitivity (\%) & Specificity (\%) & PPV (\%) & NPV (\%) & Overall agreement & Cohen's kappa \\
\hline NC-stat & $50(30-70)$ & $76(68-84)$ & $30(21-42)$ & $88(83-92)$ & $72(64-79)$ & 0.209 \\
Monofilament & $46(27-67)$ & $69(60-77)$ & $23(16-33)$ & $86(81-90)$ & $65(57-72)$ & 0.105 \\
VPT & $62(41-80)$ & $75(66-82)$ & $33(25-43)$ & $91(85-94)$ & $73(65-80)$ & 0.272 \\
Ipswich touch test & $39(20-60)$ & $85(78-91)$ & $35(22-50)$ & $87(83-90)$ & $77(70-84)$ & 0.225 \\
NDS SMALL & $89(70-98)$ & $73(65-81)$ & $40(33-48)$ & $97(91-99)$ & $76(68-82)$ & 0.418 \\
NDS LARGE & $65(44-83)$ & $41(32-50)$ & $19(14-24)$ & $85(77-91)$ & $45(37-53)$ & 0.031 \\
NDS & $54(33-73)$ & $70(61-78)$ & $27(19-37)$ & $88(83-92)$ & $67(59-75)$ & 0.171 \\
\hline
\end{tabular}

$N D S$ neuropathy disability score, $N P V$ negative predictive value, $P P V$ positive predictive value, $V P T$ vibration perception threshold

France) for CAN (defined as $\geq 1$ abnormal out of the 5 CARTs originally proposed by Ewing) was $65 \%$ and $85 \%$, respectively [25]. In another study [24], Sudoscan yielded high sensitivity $(92 \%)$ and moderate specificity (49\%) for CAN diagnosis (defined as one abnormal out of three CARTs) among 232 T2DM participants. Casellini et al. [26] studied the Spearman correlation coefficient of Sudoscan with three Ewing CARTs and with the Fourier-based method for the spectral analysis of heart rate variability. However, sensitivity, specificity and the NPV of the method were not evaluated [26]. Recently, Sudoscan reached a sensitivity of $83 \%$ and a specificity of $67 \%$ for confirmed CAN diagnosis (defined as $\geq 2$ out of 4 abnormal CARTs) among 102 individuals with diabetes (T1DM and T2DM) [27]. This finding confirmed previously published results among 75 T2DM Chinese individuals of adequate sensitivities and specificities of application of Sudoscan for hands (sensitivity $76.7 \%$ and specificity $75.6 \%$ s) and feet (sensitivity $80 \%$ and specificity $60 \%$ ) in the screening of diabetic CAN [28].

One of the leading studies in this context was published by Liatis et al. in 2007 [29]. This evaluated the indicator test for sudomotor function Neuropad (TrigoCare GmbH, Wiehl, Drabenderhöhe, Germany). The authors assessed the diagnostic utility of the latter for CAN diagnosis (defined as two abnormal out of four CARTs) in 108 T2DM and 9 T1DM human participants [29]. The diagnostic performance of Neuropad for CAN was moderate (sensitivity $59.1 \%$, specificity $46.5 \%$ ). However, its NPV was very high (92.1\%) with high sensitivity $(80.9 \%)$ and moderate specificity (50\%) compared with severe CAN [29].

Recently, in a study of 154 T2DM subjects, the use of Neuropad was associated with a high sensitivity $(70.1 \%)$ for the diagnosis of CAN (defined as at least one abnormal out of three CARTs) and high PPV (72.1\%) but with a very low NPV [30]. In this study, the heart rate variation during deep breathing and the Valsalva test were associated with parasympathetic nervous system impairment. Compared with these, Neuropad exhibited a good diagnostic performance (sensitivity 76-78\%, specificity 35-40\%, NPV 70-82\%) [30].

In a similar study of 265 T2DM participants [31], abnormal sudomotor function, as evaluated by Sudoscan, was significantly correlated only with orthostatic hypotension. On the contrary, no association was detected with the three CARTs related to heart rate variability.

Importantly, our study differs from those mentioned above in terms of design. Indeed, we did not compare the diagnostic utility of a method assessing the sympathetic nervous system in the lower extremities with the gold standard for CAN diagnosis. Instead, we evaluated a variety of simple available DPN tools, which separately evaluate large and small nerve 
fibres, and we sought to define their diagnostic performance for CAN.

The present work has its limitations. First, we did not distinguish between different degrees of CAN severity. Instead, we classified CAN as present/absent, attempting a simplification of its diagnosis. The lack of prospective data represents an additional limitation, but this was beyond the scope of this work. Moreover, the tertiary health care centre suggests that caution is required before applying our findings in the general diabetic population. Finally, during separate evaluation of the diagnostic utility of each CART and each DPN diagnostic tool, subanalysis of data was required, which unavoidably led to a smaller sample size.

The practical implications of the present study may be outlined as follows. Due to its high NPV, simple assessment of small nerve fibre function can be used as a screening tool to exclude CAN. Moreover, VPT $\geq 16$ volts may exclude CAN. Thus, CAN might be more easily excluded thanks to the use of these simpler, quicker and more widely available tools. Arguably, through this exclusion of CAN we might save time and resources by reserving further demanding and expensive diagnostic tests for humans with positive screening results. Nonetheless, it is premature to suggest that such simple tests could currently replace the established diagnosis of CAN. Furthermore, our approach offers no information on CAN severity.

\section{CONCLUSIONS}

This study has shown that assessment of small nerve fibre function by NDS (pinprick sensation, temperature perception) is the best DPN tool to exclude CAN in T2DM. This is followed by VPT. These encouraging results warrant further evaluation to facilitate wider screening for CAN, which remains a hugely under-diagnosed complication of diabetes $[2,8]$.

\section{ACKNOWLEDGEMENTS}

We thank the participants of this study.
Funding. No funding or sponsorship was received for this study or publication of this article.

Authorship. All named authors meet the International Committee of Medical Journal Editors (ICMJE) criteria for authorship for this article, take responsibility for the integrity of the work as a whole, and have given their approval for this version to be published.

Disclosures. Nikolaos Papanas is a member of the journal's Editorial Board; has been an advisory board member of Astra-Zeneca, Boehringer Ingelheim, MSD, Novo Nordisk, Pfizer, Takeda and TrigoCare International; has participated in sponsored studies by Astra-Zeneca, Eli-Lilly, GSK, MSD, Novo Nordisk, Novartis and Sanofi-Aventis; has received honoraria as a speaker for Astra-Zeneca, Boehringer Ingelheim, Eli-Lilly, Elpen, MSD, Mylan, Novo Nordisk, Pfizer, Sanofi-Aventis and Vianex; and attended conferences sponsored by TrigoCare International, Eli-Lilly, Galenica, Novo Nordisk, Pfizer and Sanofi-Aventis. Kalliopi Pafili's current affiliation is Institute for Clinical Diabetology, German Diabetes Centre, Leibniz Institute for Diabetes Research at Heinrich-Heine University, 40225, Düsseldorf, Germany but she retains an adjunct appointment at Diabetes Centre, Second Department of Internal Medicine, Democritus University of Thrace, University Hospital of Alexandroupolis, Greece. Grigorios Trypsianis, Dimitrios Papazoglou and Efstratios Maltezos have nothing to disclose.

Compliance with Ethics Guidelines. This study received approval from the institutional ethics committee. The study was conducted in accordance with the Declaration of Helsinki 1964 and its later amendments. Informed consent was obtained from all participants of this study.

Data Availability. The datasets during and/ or analyzed during the current study are available from the corresponding author on reasonable request. 
Open Access. This article is licensed under a Creative Commons Attribution-NonCommercial 4.0 International License, which permits any non-commercial use, sharing, adaptation, distribution and reproduction in any medium or format, as long as you give appropriate credit to the original author(s) and the source, provide a link to the Creative Commons licence, and indicate if changes were made. The images or other third party material in this article are included in the article's Creative Commons licence, unless indicated otherwise in a credit line to the material. If material is not included in the article's Creative Commons licence and your intended use is not permitted by statutory regulation or exceeds the permitted use, you will need to obtain permission directly from the copyright holder. To view a copy of this licence, visit http://creativecommons.org/licenses/by$\mathrm{nc} / 4.0 /$.

\section{REFERENCES}

1. Standards of medical care in diabetes-2017: summary of revisions. Diabetes Care. 2017; 40:S4-S5.

2. Spallone V, Ziegler D, Freeman R, et al. Cardiovascular autonomic neuropathy in diabetes: clinical impact, assessment, diagnosis, and management. Diabetes Metab Res Rev. 2011;27:639-53.

3. Ewing DJ, Martyn CN, Young RJ, Clarke BF. The value of cardiovascular autonomic function tests: 10 years experience in diabetes. Diabetes Care. 1985;8:491-8.

4. Körei AE, Kempler M, Istenes I, et al. Why not to use the handgrip test in the assessment of cardiovascular autonomic neuropathy among patients with diabetes mellitus? Curr Vasc Pharmacol. 2017;15: 66-73.

5. Pafili K, Trypsianis G, Papazoglou D, Maltezos E, Papanas N. Simplified diagnosis of cardiovascular autonomic neuropathy in type 2 diabetes using Ewing's battery. Rev Diabet Stud. 2015;12:213-9.

6. Stranieri A, Abawajy J, Kelarev A, Huda S, Chowdhury M, Jelinek HF. An approach for Ewing test selection to support the clinical assessment of cardiac autonomic neuropathy. Artif Intell Med. 2013;58:185-93.
7. Bellavere F, Ragazzi E, Chilelli NC, Lapolla A, Bax G. Autonomic testing: which value for each cardiovascular test? An observational study. Acta Diabetol. 2019;56:39-43.

8. Pafili K, Papanas N. Diagnosis of cardiovascular autonomic neuropathy in diabetes: as simple as possible, but not simpler? Acta Diabetol. 2019;56: 601-2.

9. Migdalis I, Czupryniak L, Lalic N, Leslie RD, Papanas N, Valensi P. Diabetic microvascular complications. Int J Endocrinol. 2018;2018:5683287.

10. Bönhof GJ, Herder C, Strom A, Papanas N, Roden M, Ziegler D. Emerging biomarkers, tools, and treatments for diabetic polyneuropathy. Endocr Rev. 2019;40:153-92.

11. Papanas N, Ziegler D. New vistas in the diagnosis of diabetic polyneuropathy. Endocrine. 2014;47: 690-8.

12. Pafili K, Trypsianis G, Papazoglou D, Maltezos E, Papanas N. Cardiovascular autonomic neuropathy and distal symmetric sensorimotor polyneuropathy: these two diabetic microvascular complications do not invariably co-exist. Curr Vasc Pharmacol. 2020;18:50-6.

13. Pafili K, Maltezos E, Papanas N. NC-stat for the diagnosis of diabetic polyneuropathy. Expert Rev Med Devices. 2017;14:251-4.

14. Chatzikosma G, Pafili K, Demetriou M, Vadikolias K, Maltezos E, Papanas N. Evaluation of sural nerve automated nerve conduction study in the diagnosis of peripheral neuropathy in patients with type 2 diabetes mellitus. Arch Med Sci. 2016;12:390-3.

15. Pafili K, Papanas N, Ziegler D. Neuropathy in diabetes: "One cannot begin it too soon". Angiology. 2018;69:752-4.

16. Pop-Busui R, Boulton AJ, Feldman EL, et al. Diabetic neuropathy: a position statement by the American Diabetes Association. Diabetes Care. 2017;40: 136-54.

17. Young MJ, Breddy JL, Veves A, Boulton AJ. The prediction of diabetic neuropathic foot ulceration using vibration perception thresholds. A prospective study. Diabetes Care. 1994;17:557-60.

18. An experience with the use of biothesiometer in diabetics at a tertiary care centre. Available: http:// www.pjmhsonline.com/2015/jan_march/pdf/423\% 20An\%20Experience\%20with\%20the\%20Use \%20of \%20Biothesiometer\%20in\%20Diabetics\%20at\%20a\% 20Tertiary\%20Care\%20Centre.pdf. Accessed 13 June 2019. 
19. Amin N, Doupis J. Diabetic foot disease: from the evaluation of the "foot at risk" to the novel diabetic ulcer treatment modalities. World J Diabetes. 2016;7:153-64.

20. Baraz S, Zarea K, Shahbazian HB, Latifi SM. Comparison of the accuracy of monofilament testing at various points of feet in peripheral diabetic neuropathy screening. J Diabetes Metab Disord. 2014;13:19.

21. Rayman G, Vas PR, Baker $\mathrm{N}$, et al. The Ipswich Touch Test: a simple and novel method to identify in patients with diabetes at risk of foot ulceration. Diabetes Care. 2011;34:1517-8.

22. Manes C, Papanas N, Exiara T, et al. The indicator test Neuropad in the assessment of small and overall nerve fibre dysfunction in patients with type 2 diabetes: a large multicentre study. Exp Clin Endocrinol Diabetes. 2014;122:195-9.

23. Young MJ, Boulton AJ, MacLeod AF, Williams DR, Sonksen PH. A multicentre study of the prevalence of diabetic peripheral neuropathy in the United Kingdom hospital clinic population. Diabetologia. 1993;36:150-4.

24. Yajnik CS, Kantikar V, Pande A, et al. Screening of cardiovascular autonomic neuropathy in patients with diabetes using non-invasive quick and simple assessment of sudomotor function. Diabetes Metab. 2013;39:126-31.

25. Selvarajah D, Cash T, Davies J, et al. SUDOSCAN: a simple, rapid, and objective method with potential for screening for diabetic peripheral neuropathy. PLoS One. 2015;10:e0138224.
26. Casellini CM, Parson HK, Richardson MS, Nevoret ML, Vinik AI. Sudoscan, a noninvasive tool for detecting diabetic small fiber neuropathy and autonomic dysfunction. Diabetes Technol Ther. 2013;15:948-53.

27. D'Amato C, Greco C, Lombardo G, et al. The diagnostic usefulness of the combined COMPASS 31 questionnaire and electrochemical skin conductance for diabetic cardiovascular autonomic neuropathy and diabetic polyneuropathy. J Peripher Nerv Syst. 2020. (Epub ahead of print).

28. He T, Wang C, Zuo A, et al. Electrochemical skin conductance may be used to screen for diabetic cardiac autonomic neuropathy in a Chinese population with diabetes. J Diabetes Res. 2017;2017: 8289740 .

29. Liatis S, Marinou K, Tentolouris N, Pagoni S, Katsilambros $\mathrm{N}$. Usefulness of a new indicator test for the diagnosis of peripheral and autonomic neuropathy in patients with diabetes mellitus. Diabet Med. 2007;24:1375-80.

30. Mendivil CO, Kattah W, Orduz A, Tique C, Cárdenas JL, Patiño JE. Neuropad for the detection of cardiovascular autonomic neuropathy in patients with type 2 diabetes. J Diabetes Complicat. 2016;30: 93-8.

31. Yajnik CS, Kantikar VV, Pande AJ, Deslypere JP. Quick and simple evaluation of sudomotor function for screening of diabetic neuropathy. ISRN Endocrinol. 2012;2012:103714. 